\title{
Multilocus sequence typing of Enterocytozoon bieneusi in crab-eating macaques (Macaca fascicularis) in Hainan, China
}

Li Chen ${ }^{1}$, Na Li ${ }^{2,3}$, Yaqiong Guo ${ }^{2,3}$, Jianguo Zhao ${ }^{4}$, Yaoyu Feng ${ }^{1,2,3^{*}}$ and Lihua Xiao ${ }^{2,3^{*}}$ (1)

\begin{abstract}
Background: Enterocytozoon bieneusi is one of common intestinal pathogens in humans and animals including nonhuman primates (NHPs). Many zoonotic pathogens including E. bieneusi have been found in these animals. However, there are few studies on the population structure of E. bieneusi in NHPs. To infer the gene diversity and population genetics of E. bieneusi, we selected 88 E. bieneusi-positive samples from crab-eating macaques for multilocus characterizations in this study.

Methods: The E. bieneusi isolates examined belonged to three common genotypes with different host ranges by sequence analysis of the ribosomal internal transcribed spacer (ITS): Type IV $(n=44)$, Macaque3 $(n=24)$ and Peru8 $(n=20)$. They were further characterized by sequence analysis at four microsatellite and minisatellite loci (MS1, MS3, MS4 and MS7). DnaSP, Arlequin and LIAN were used to analyze the sequence data together with those from the ITS locus to infer the population genetics. Subpopulation structure was inferred using phylogenetic and STRUCTURE analyses.
\end{abstract}

Results: Seventy-two (81.8\%), 71 (80.7\%), 76 (86.4\%) and 79 (89.8\%) samples were amplified and sequenced successfully at the MS1, MS3, MS4 and MS7 loci, respectively, with 53 having sequence data at all five MLST loci including ITS. Altogether, 33 multilocus genotypes (MLGs) were produced based on concatenated sequences from the 53 samples. In phylogenetic analyses of sequences and allelic data, four major subpopulations (SPs) were observed with different ITS genotypes in each of them: Type IV and Peru8 in SP1 and SP2; Type IV, Macaque3 and Peru8 in SP3; and Type IV and Macaque3 in SP4. SP3 and SP4 were phylogenetically related and might be NHP-specific based on the fact that Macaque3 is mostly found in NHPs. A strong linkage disequilibrium (LD) was observed among the multilocus sequences and allelic data.

Conclusions: The significant LD in the multilocus sequence analysis indicated the presence of an overall clonal population structure of E. bieneusi in crab-eating macaques. The inconsistent segregation of MLGs among ITS genotypes

\footnotetext{
*Correspondence: yyfeng@ecust.edu.cn; Ixiao1961@gmail.com

1 State Key Laboratory of Bioreactor Engineering, School of Resource

and Environmental Engineering, East China University of Science and Technology, Shanghai 200237, China

${ }^{2}$ Key Laboratory of Zoonosis of Ministry of Agriculture, College

of Veterinary Medicine, South China Agricultural University,

Guangzhou 510642, China

Full list of author information is available at the end of the article
}

\section{$\triangle B M C$}

Author(s) 2020. This article is licensed under a Creative Commons Attribution 4.0 International License, which permits use, sharing adaptation, distribution and reproduction in any medium or format, as long as you give appropriate credit to the original author(s) and the source, provide a link to the Creative Commons licence, and indicate if changes were made. The images or other third party material in this article are included in the article's Creative Commons licence, unless indicated otherwise in a credit line to the material. If material is not included in the article's Creative Commons licence and your intended use is not permitted by statutory regulation or exceeds the permitted use, you will need to obtain permission directly from the copyright holder. To view a copy of this licence, visit http://creativecommons.org/licenses/by/4.0/. The Creative Commons Public Domain Dedication waiver (http://creativecommons.org/publicdomain/zero/1.0/) applies to the data made available in this article, unless otherwise stated in a credit line to the data. 
suggested some occurrence of genetic recombination. These observations should improve our understanding of the population genetics of E. bieneusi in NHPs.

Keywords: Enterocytozoon bieneusi, Multilocus sequence typing, Crab-eating macaques, Population structure, Subpopulation

\section{Background}

Enterocytozoon bieneusi is an obligate intracellular pathogen infecting humans, domestic animals and wildlife [1]. Since the detection of E. bieneusi in AIDS patients in 1985 for the first time, this pathogen has been found in other immunocompromised/immunodeficient individuals, such as organ transplant recipients, children, and lifethreatening and persistent diarrhea often occur in these populations [2-7]. It has been recently identified in several species of non-human primates (NHPs) in China and elsewhere [8-14].

Approximately 500 E. bieneusi genotypes have been identified based on sequence analysis of the internal transcribed spacer (ITS) region of the rRNA gene $[15,16]$. They belong to 11 phylogenetic groups. Among them, ITS genotypes in Group 1 and Group 2 have been found in a broad range of hosts including humans and are probably responsible for most zoonotic or cross-species $E$. bieneusi infections whereas host adaptation seems to be more common in genotypes of Groups 3 to 11 according to a recent review of E. bieneusi [6]. To date, more than 100 E. bieneusi genotypes have been detected in NHPs, mostly belonging to Group $1[6,9,10,13,14,17-21]$. Among them, Type IV and Peru8 are common ITS genotypes in humans and various animals in many countries, including China [22]. Conversely, Macaque3 has been mostly found in monkeys and occasionally in dogs in China [1, 21, 23-26].

Multilocus sequence typing (MLST) based on sequence analysis of four markers (MS1, MS3, MS4 and MS7) with simple tandem repeats and the ITS has been used in investigating the transmission dynamics of $E$. bieneusi in humans and some animals, including pigs, sheep, rabbits, calves, black bears, giant pandas and horses [27-35]. Thus far, only three studies have used the MLST analysis to characterize E. bieneusi isolates in NHPs, and only one study has found the presence of an overall clonal population structure and an epidemic population structure in subpopulations of this pathogen in NHPs [11, 36, 37].

In this study, 88 E. bieneusi-positive samples from crab-eating macaques (Macaca fascicularis) in Hainan Province, China were used for MLST analysis and population genetic characterization of $E$. bieneusi. The present study aims at evaluating the genetic diversity and population structure of E. bieneusi in NHPs to better understand the transmission and epidemiology of $E$. bieneusi in these animals.

\section{Methods \\ Source of $E$. bieneusi-positive samples}

The 88 E. bieneusi-positive samples used in this study were from crab-eating macaques in Hainan, China. They consisted of ITS genotypes Type IV $(n=44)$, Macaque3 $(n=24)$ and Peru8 $(n=20)$, which were identified by sequence analysis of the ITS region of the rRNA gene in a previous study [8]. They were collected from different animals and used for further molecular characterization in this study. Other minor E. bieneusi genotypes from that study were not included in the present study, as the small numbers of samples for each of them made examinations of intra-genotypic variations of them difficult.

\section{MLST analysis of E. bieneusi}

Four established MLST loci were targeted in this study, including three microsatellite loci (MS1, MS3 and MS7) and one minisatellite locus (MS4). These loci were amplified by nested PCR using published primers and conditions [32]. A positive control (DNA of genotype BEB4 which usually infects cattle rather than NHPs) and a negative control (reagent water without DNA) were used in all PCR tests. PCR was performed twice on each DNA sample at each genetic locus. The secondary PCR products generated were bi-directionally sequenced on an ABI 3730 Genetic Analyzer (Applied Biosystems, Foster City, CA). The nucleotide sequences obtained were assembled using software ChromasPro 1.32 (http://technelysium.com.au/ChromasPro.html), and aligned with each other and homologous sequences downloaded from GenBank using ClustalX (http://clust al.org). The sequence alignments at the four genetic loci (MS1, MS3, MS4 and MS7) were used in the assessment of sequence polymorphism within the ITS genotypes, determination of MLGs and phylogenetic analyses of the MLST data.

\section{Population genetic analysis of sequence data}

The multilocus sequences data (concatenated sequences from all five markers, including the ITS) generated were used in the analysis of the population genetics of 
E. bieneusi using the software DnaSP version 5.10.01 (http://www.ub.edu/dnasp/) [38]. The population structure of E. bieneusi was assessed by intragenic and intergenic linkage disequilibrium (LD), neutrality (Fu's statistics or Fs values on selective neutrality based on genotype frequency), and recombination events (Rms).

\section{Population genetic analysis of allelic profile data}

Pairwise intergenic LD based on allelic profiles at the five genetic loci was evaluated using the exact test and Markov chain parameters implemented in Arlequin version 3.5.1.2 [39]. The standardized index of association $\left(I_{\mathrm{A}}^{\mathrm{S}}\right)$ was measured using LIAN $3.7[40]$.

\section{Subpopulation structure analysis}

The phylogenetic relationship among MLGs including both SNPs and INDELs was assessed using the maximum likelihood (ML) analysis and general time reversible model implemented in MEGA 6 (https://www. megasoftware.net). The reliability of clusters formed was assessed using bootstrap analysis with 1000 replicates. An un-weighted pair group mean average (UPGMA) tree of the allelic data was generated using MEGA 6. Wright's fixation index $\left(F_{\mathrm{ST}}\right)$ of $E$. bieneusi isolates was calculated using software Arlequin version 3.5.2.2.

STRUCTURE version 2.3.1 was used to identify distinct sub-populations, with $K$-values of 2-4 [41]. $F_{\mathrm{ST}}$ was calculated to assess the robustness of the substructuring and to determine genetic distances between subpopulations.

\section{Results}

\section{PCR amplification efficiency at the MLST loci}

The PCR amplification efficiencies were $81.8 \%, 80.7 \%$, $86.4 \%$ and $89.8 \%$ at the MS1, MS3, MS4 and MS7 loci, respectively (Table 1 ). For ITS genotypes Type IV and Peru8, the amplification efficiencies were similar at the MS1 (88.7\% and $80.0 \%)$, MS3 (81.8\% and $85.0 \%)$, MS4 (86.4\% and $85.0 \%$ ) and MS7 (91.2\% and 95.0\%) loci. For Macaque3, however, the amplification efficiencies at

Table 1 PCR amplification efficiency of DNA from four Enterocytozoon bieneusi ITS genotypes at the other four MLST loci

\begin{tabular}{llllll}
\hline ITS genotype $n$ & $n \begin{array}{l}\text { No. of specimens amplified/No. of specimens } \\
\text { analyzed (\%) }\end{array}$ \\
\cline { 2 - 6 } & & MS1 & MS3 & MS4 & MS7 \\
\hline Type IV & 44 & $39 / 44(88.7)$ & $36 / 44(81.8)$ & $38 / 44(86.4)$ & $41 / 44(91.2)$ \\
Macaque3 & 24 & $17 / 24(70.8)$ & $18 / 24(75.0)$ & $21 / 24(87.5)$ & $19 / 24(79.2)$ \\
Peru8 & 20 & $16 / 20(80.0)$ & $17 / 20(85.0)$ & $17 / 20(85.0)$ & $19 / 20(95.0)$ \\
Total & 88 & $72 / 88(81.8)$ & $71 / 88(80.7)$ & $76 / 88(86.4)$ & $79 / 88(89.8)$
\end{tabular}

Abbreviation: $\mathrm{n}$, number of samples the MS1 (70.8\%), MS3 (75.0\%), MS4 (87.5\%) and MS7 (79.2\%) loci were correspondingly lower than those of ITS genotypes Type IV and Peru8 at the three loci except MS4.

\section{Multilocus genotypes}

Genetic diversity at the MLST loci was in the form of both SNPs and indels, with 24, 5, 25, 4 and 3 segregation sites at the MS1, MS3, MS4, MS7 and ITS loci, respectively (Table 2). Altogether, 6, 4, 6, 2 genotypes were detected at the MS1, MS3, MS4 and MS7 loci, respectively. The intragenic LD among segregating sites for each locus was calculated using the linear regression equation. The MS3, MS7 and ITS loci were in complete linkage disequilibrium $(L D=1)$. In contrast, the remaining two loci (MS1 and MS4) had incomplete LD, indicating the potential presence of genetic recombination. This was confirmed by further assessment of intragenic recombination, which showed the presence of two and five Rms at the MS1 and MS4 loci, respectively.

Of the 88 samples analyzed by MLST, 53 were positive at all four genetic loci. To determine the MLGs, sequences of the five genetic loci (MS1, MS3, MS4, MS7 and ITS) from each of the 53 samples were concatenated. Genetic diversity was assessed based on both total polymorphic sites (finite population variance estimates) and segregating sites only (infinite population variance estimates). Together they formed 33 MLGs with an Hd of 0.98 (Table 3). The mean numbers of pairwise differences in polymorphic and segregating sites were 16 and 14 , respectively. The frequency of the MLGs ranged from 9.4\% (one MLG with five samples), 7.5\% (four MLGs each with three samples), 3.8\% (eight MLGs each with two samples), to $1.9 \%$ (20 MLGs each with one sample) (Table 4).

\section{Population structure}

Inter-locus LD over all segregating sites within the multilocus contig was assessed for all 53 samples. This produced a $Z_{\mathrm{n}} s$ value of 0.185 ; the probability $P$-values for expected $Z_{\mathrm{n}} s \leq 0.185$ was 0.976 . The incomplete LD among these 53 samples $\left(\left|D^{\prime}\right| \mathrm{Y}=0.8679-0.2781 \mathrm{X}\right)$ indicated a possible occurrence of genetic recombination, which was confirmed by results of the recombination analysis (10 Rms) (Table 3). Thereafter, we used Fu's statistic test to evaluate the genotype frequency in this population. In this test, we obtained Fs values of $-5.56(P$ $(\mathrm{Fs}<-5.56)=0.158)$ and $-6.91(P(\mathrm{Fs}<-6.91)=0.122)$ based on polymorphic sites and segregating sites, respectively (Table 3). The results of Fu's statistic tests indicated the presence of an excessive number of alleles.

We calculated $I_{\mathrm{A}}^{\mathrm{S}}$ to assess the LD between alleles in pairwise combinations of genetic loci [39]. Results of this 
Table 2 Intragenic linkage disequilibrium and recombination events in individual genetic loci among 53 E. bieneusi samples

\begin{tabular}{|c|c|c|c|c|c|c|c|}
\hline Marker & No. of types & S & $P$ & $\mathrm{~F}$ & B & LD (|D१) & $\mathrm{Rm}$ \\
\hline MS1 & 6 & 24 & 253 & 3 & 0 & $Y=0.9838+0.0293 X$ & 2 \\
\hline MS3 & 4 & 5 & 10 & 0 & 0 & 1 & 0 \\
\hline MS4 & 6 & 25 & 276 & 0 & 0 & $Y=0.7859+0.0126 X$ & 5 \\
\hline MS7 & 2 & 4 & 6 & 0 & 0 & 1 & 0 \\
\hline ITS & 3 & 3 & 3 & 0 & 0 & 1 & 0 \\
\hline
\end{tabular}

Abbreviations: $S$, number of segregating sites; $P$, number of pairwise comparisons; F, number of significant pairwise comparisons by Fisher's exact test; $B$, number of significant comparisons after Bonferroni correction; LD $(|D|)$, linkage disequilibrium per site; Rm, minimum number of recombination events

Table 3 Genetic diversity in 53 Enterocytozoon bieneusi samples based on the analysis of concatenated multilocus sequences (2661 bp in length)

\begin{tabular}{|c|c|c|c|c|c|c|c|c|c|c|c|}
\hline \multirow[t]{2}{*}{ Test model } & \multicolumn{11}{|c|}{ Variability of multilocus sequences } \\
\hline & No. MLGs & $\mathrm{Hd}$ & k & $\mathrm{Pi}$ & Theta $(\mathrm{k})$ & Fs (obs) & $P($ Fs $\leq \mathrm{obs})$ & $Z_{n} s$ (obs) & $P\left(Z_{n} s \leq o b s\right)$ & $\operatorname{LD}(\mid D \uparrow)$ & $\mathrm{Rm}$ \\
\hline Polymorphic sites & 33 & 0.98 & 16 & 0.009 & 16 & -5.56 & 0.158 & & & & \\
\hline Segregating sites & 33 & 0.98 & 14 & 0.008 & 14 & -6.91 & 0.122 & 0.185 & 0.976 & $Y=0.8679-0.2781 X$ & 10 \\
\hline
\end{tabular}

Abbreviations: MLG, multilocus genotype; Hd, gene diversity; $\mathrm{k}$, mean number of pairwise differences; Pi, nucleotide diversity (average over loci); theta (k), genetic variance based on the mean number of pairwise differences; $F s$, Fu's statistic testing selective neutrality based on genotype frequency; Obs, observed value; $P$ (Fs $\leq$ Obs), probability of obtaining Fs values equal or lower than the observed; $Z_{n} s$ (obs), observed $Z_{n} s$ value; $P\left(Z_{n} s \leq\right.$ Obs), probability of obtaining $Z_{n} s$ values equal or lower than the observed; $L D\left(\left|D^{\prime}\right|\right)$, linkage disequilibrium per site; $R m$, minimum number of recombination events

analysis of allelic profile data showed a positive $I_{\mathrm{A}}^{\mathrm{S}}$ value together with $V_{D}>L$. Furthermore, a significant $P_{M C}$ value $(<0.001)$ was obtained using the Monte Carlo method based on the null hypothesis of panmixia (Table 5). All these results indicated that the population was in LD. However, values of $V_{D}<L$ and $P_{M C}>0.001$ were obtained for subpopulations identified in this study or ITS genotype. Thus, these subpopulations and ITS genotypes were in linkage equilibrium (LE) and had the epidemic population structure.

\section{Subpopulation structure}

Maximum likelihood analysis of the concatenated sequences of the 33 MLGs showed four main subpopulations (SPs). SP1 contained samples of three MLGs of ITS genotype Type IV and two MLGs of ITS genotype Peru8, SP2 contained six MLGs of ITS genotype Type IV and two MLGs of ITS genotype Peru8, SP3 contained seven MLGs of ITS genotype Type IV, four MLGs of ITS genotype Peru8, and five MLGs of ITS genotype Macaque3, while SP4 contained two MLGs of ITS genotype Type IV and two MLGs of ITS genotype Macaque3 (Fig. 1). Similarly, four major clades were formed in UPGMA analysis of 33 MLGs in agreement with results of ML analysis of the concatenated sequence data (Fig. 2).

In STRUCTURE analysis of the MLST data, we used the smallest value of $K(K=2)$ to measure population differentiation within the E. bieneusi population. Two subpopulations (SPs) with alpha $(\alpha)$ value of 0.104 were produced (1, 2; Fig. 3a). SP1 contained 14 MLGs, while SP2 contained the remaining 19 MLGs (Fig. 3a). When the $K$ value was increased to 3 , three clear SPs were formed ( $\alpha=0.074$; Fig. 3b). SP1 included 8 MLGs belonging to SP1 at $K=2$, SP2 included 3 MLGs belonging to SP1 and 7 MLGs belonging to SP2 at $K=2$, while SP3 included 3 MLGs belonging to SP1 and 12 MLGs belonging to SP2 at $K=2$ (Fig. 3a, b). When using $K=4$, four clear SPs formed, with individuals in SP1 being similar to SP1 at $K=2$ and 3 (Fig. 3). In addition, the $\alpha$ value of 0.061 was similar to the values generated at $K=2$ and 3 , suggesting the substructures at $K=4$ could be realistic.

$F_{\mathrm{ST}}$ analysis was used to assess the sub-structuring within the E. bieneusi population. For SP1 and SP2 generated by STRUCTURE at $K=2$, the differentiation was significant $\left(F_{\mathrm{ST}}=0.161, P<0.0001\right)$. At $K=3$, the differentiation between SP1 and SP2 $\left(F_{\mathrm{ST}}=0.362, P<0.0001\right)$, SP1 and SP3 $\left(F_{\mathrm{ST}}=0.274, P<0.0001\right)$, and SP2 and SP3 $\left(F_{\mathrm{ST}}=0.234, \quad P<0.0001\right)$ were also significant. Similarly at $K=4$, the differentiation between SP1 and SP2 $\left(F_{\mathrm{ST}}=0.349, \quad P<0.0001\right), \quad \mathrm{SP} 1$ and SP3 $\quad\left(F_{\mathrm{ST}}=0.345\right.$, $P<0.0001)$, SP1 and SP4 $\left(F_{\mathrm{ST}}=0.313, P<0.0001\right), \mathrm{SP} 2$ and SP3 $\left(F_{\mathrm{ST}}=0.293, P<0.0001\right), \mathrm{SP} 2$ and SP4 $\left(F_{\mathrm{ST}}=0.233\right.$, $P<0.0001)$, and SP3 and SP4 $\left(F_{\mathrm{ST}}=0.254, P<0.0001\right)$ were all significant. 
Table 4 Multilocus sequence types of ITS genotypes Type IV and Macaque3 and Peru8 of Enterocytozoon bieneusi in crab-eating macaques

\begin{tabular}{|c|c|c|c|c|c|c|}
\hline \multirow[t]{2}{*}{ MLG } & \multirow[t]{2}{*}{ ITS genotype } & \multicolumn{4}{|c|}{ Sequence type ${ }^{\mathrm{a}}$} & \multirow{2}{*}{$\begin{array}{l}\text { No. of } \\
\text { individuals }\end{array}$} \\
\hline & & MS1 & MS3 & MS4 & MS7 & \\
\hline T1 & \multirow[t]{18}{*}{ Type IV } & 3 & 3 & 2 & 1 & 3 \\
\hline T2 & & 1 & 1 & 2 & 1 & 1 \\
\hline T3 & & 6 & 2 & 1 & 1 & 2 \\
\hline T4 & & 1 & 2 & 3 & 1 & 2 \\
\hline T5 & & 3 & 2 & 1 & 1 & 1 \\
\hline T6 & & 2 & 2 & 2 & 1 & 1 \\
\hline T7 & & 4 & 1 & 2 & 1 & 2 \\
\hline T8 & & 3 & 1 & 1 & 1 & 2 \\
\hline T9 & & 3 & 2 & 2 & 1 & 3 \\
\hline T10 & & 2 & 3 & 1 & 1 & 1 \\
\hline T11 & & 3 & 1 & 2 & 1 & 5 \\
\hline Т12 & & 4 & 2 & 2 & 1 & 1 \\
\hline Т13 & & 5 & 3 & 4 & 2 & 1 \\
\hline T14 & & 2 & 3 & 2 & 1 & 1 \\
\hline T15 & & 5 & 1 & 1 & 1 & 1 \\
\hline T16 & & 2 & 3 & 4 & 2 & 1 \\
\hline T17 & & 3 & 1 & 3 & 2 & 1 \\
\hline Т18 & & 5 & 1 & 2 & 1 & 1 \\
\hline $\mathrm{Cl}$ & \multirow[t]{7}{*}{ Macaque3 } & 2 & 3 & 2 & 2 & 2 \\
\hline$C 2$ & & 2 & 1 & 2 & 2 & 2 \\
\hline C3 & & 3 & 2 & 2 & 1 & 3 \\
\hline C4 & & 1 & 1 & 2 & 1 & 2 \\
\hline C5 & & 3 & 4 & 6 & 1 & 1 \\
\hline C6 & & 3 & 1 & 2 & 1 & 1 \\
\hline$C 7$ & & 3 & 4 & 2 & 1 & 1 \\
\hline P1 & \multirow[t]{9}{*}{ Peru8 } & 3 & 2 & 2 & 1 & 3 \\
\hline P2 & & 1 & 2 & 2 & 1 & 1 \\
\hline P3 & & 3 & 1 & 2 & 1 & 1 \\
\hline P4 & & 1 & 1 & 2 & 1 & 1 \\
\hline P5 & & 3 & 2 & 1 & 1 & 2 \\
\hline P6 & & 2 & 2 & 1 & 1 & 1 \\
\hline P7 & & 2 & 4 & 5 & 2 & 1 \\
\hline P8 & & 3 & 4 & 5 & 1 & 1 \\
\hline Total & & - & - & - & - & 53 \\
\hline
\end{tabular}

${ }^{a}$ All four MLST loci amplified successfully were taken into consideration

\section{Discussion}

Currently, multilocus sequence typing has been used to analyze the genetic diversity of $E$. bieneusi around the world [15, 42, 43]. In Peru, India and Nigeria, 66 MLGs were identified in HIV-infected individuals [33, 37]. In China, 59 and 44 MLGs were identified in NHPs and pigs, respectively, while some genetic diversity based on MLST data was also observed in E. bieneusi isolates from domestic, wild, and zoo animals [29, 36, 42-48]. However, few studies analyzed the MLGs of this pathogen in
NHPs in China. In the present study, 33 MLGs were identified from $53 \mathrm{E}$. bieneusi-positive samples from macaque monkeys, indicating a high genetic diversity within some common ITS genotypes (Type IV, Macaques3 and Peru8), and supporting high-resolution of the multilocus sequencing tool established by Feng et al. [32].

A strong and significant intragenic LD was observed in the analysis of the MLST data, indicating an overall clonal population structure of E. bieneusi in this study. In previous studies, the strong and significant intragenic 
Table 5 Results of linkage disequilibrium analysis of allelic profile data

\begin{tabular}{llllllll}
\hline Population $^{\mathrm{a}}$ & $n$ & $H$ & $P_{A}$ & $P_{M C}$ & $V_{D}$ & $L$ \\
\hline All & 33 & $0.6042 \pm 0.1024$ & 0.0781 & $<0.001$ & 1.0253 & 0.9422 \\
SP 1 & 5 & $0.5600 \pm 0.1600$ & 0.0432 & 0.487 & 0.8444 & 1.1362 \\
SP 2 & 9 & $0.4667 \pm 0.1325$ & -0.0741 & 0.908 & 0.6286 & 1.3191 & $\mathrm{~N}$ \\
SP 3 & 15 & $0.4549 \pm 0.1400$ & 0.0126 & 0.604 & 0.8904 & 1.0392 & $\mathrm{~N}$ \\
SP 4 & 4 & $0.3200 \pm 0.1497$ & -0.0590 & 1.000 & 0.4889 & 1.0765 & $\mathrm{~N}$ \\
Type IV & 18 & $0.5020 \pm 0.1542$ & 0.0394 & 0.319 & 0.8962 & 0.9065 & $\mathrm{~N}$ \\
Macaque3 & 7 & $0.4476 \pm 0.1425$ & 0.0484 & 0.365 & 0.9905 & 1.2423 & $\mathrm{~N}$ \\
Peru8 & 8 & $0.4786 \pm 0.1497$ & 0.0820 & 0.044 & 1.0622 & 1.1025 & $\mathrm{~N}$ \\
\hline
\end{tabular}

a Considering each group of isolates with the same multilocus genotype (MLG) as one individual

Abbreviations: $n$, number of isolates; $\mathrm{H}$, mean genetic diversity; $I_{A}^{S}$, standardized index of association calculated using the program LIAN 3.7; $P_{M C}$, significance for obtaining this value in 1000 simulations using the Monte Carlo method; $V_{D}$, variance of pairwise differences; $L, 95 \%$ critical value for $V_{D} ; V_{D}>L$ indicates linkage disequilibrium; $\mathrm{Y}$, yes; $\mathrm{N}$, no

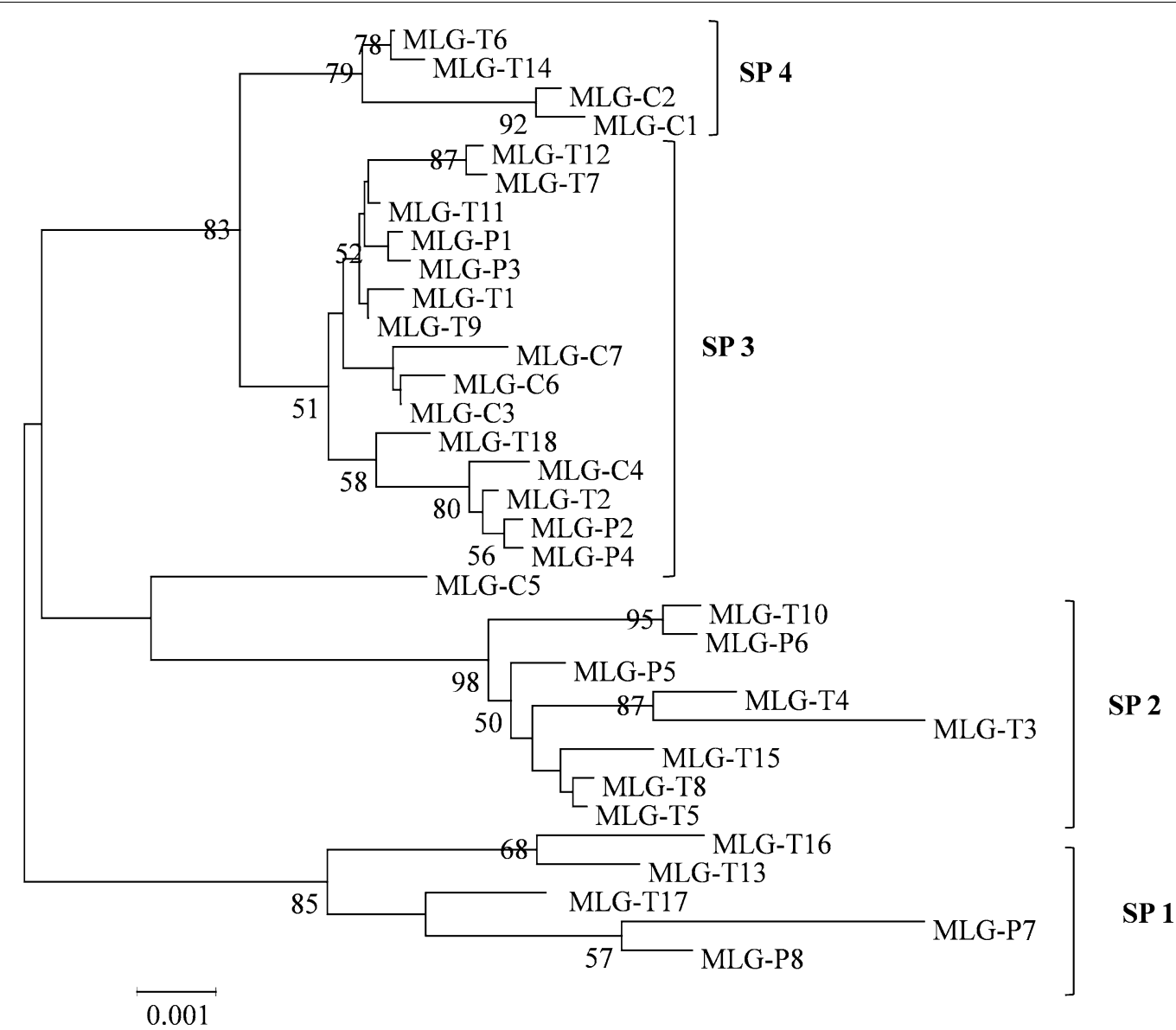

Fig. 1 Phylogenetic relationships of multilocus genotypes (MLGs) of ITS genotypes Type IV, Macaque3 and Peru8 of Enterocytozoon bieneusi inferred using the maximum likelihood analysis of concatenated ITS, MS1, MS3, MS4 and MS7. Bootstrap values greater than 50\% from 1000 replicates are shown at the nodes 

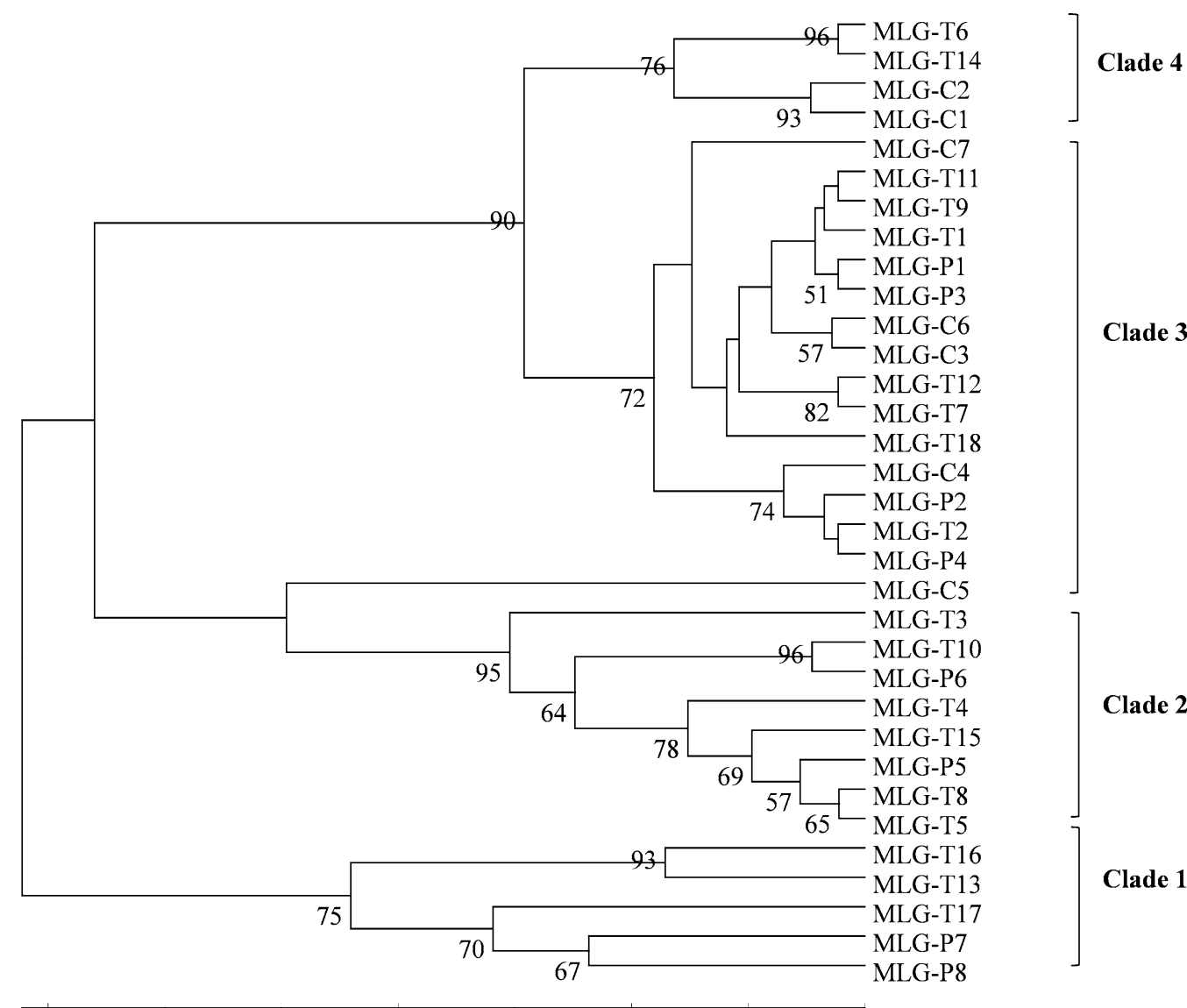

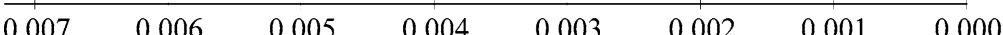

Fig. 2 An unweighted pair-group mean average (UPGMA) tree for multilocus genotypes (MLGs) of ITS genotypes Type IV, Macaque3 and Peru8 of Enterocytozoon bieneusi. Bootstrap values greater than 50\% from 1000 replicates are shown at the nodes

LD obtained was used as an indication for the presence of a clonal population structure of E. bieneusi in the AIDS patients, giant pandas, pigs, fur animals and NHPs [36, $37,47,49,50]$. Therefore, there was a clonal endemicity of $E$. bieneusi in the study population.

Nevertheless, the MLGs found in crab-eating macaques were not segregated by ITS genotypes, indicating the existence of genetic recombination in the E. bieneusi population studied. In previous investigations, E. bieneusi MLGs were segregated by ITS genotypes for some genotypes with narrower host ranges, such as A, EbpA, SCO2 and Cs-4, but not for zoonotic genotypes such as Type IV, D, WL11 and Peru11 $[15,16]$. Similarly, in this study, MLGs of E. bieneusi in crab-eating macaques were also not segregated by ITS genotypes, especially for the zoonotic genotypes Type IV and Peru8. Genetic recombination appears to be common in some microsporidia of insects, including Kneallhazia solenopsae in fire ants and Andreanna caspii in mosquitoes. In a previous study in China, the MLGs of some common ITS genotypes (Type
IV, Peru8, D, Macaque3, CM2, Peru11, Henan V, PigEBITS7) in NHPs were not segregated by ITS genotypes in phylogenetic analysis, suggesting a possible occurrence of sexual recombination in E. bieneusi [36]. Therefore, data from both studies have indicated that there could be some sexual recombination among common E. bieneusi genotypes in NHPs.

Four SPs were obtained in this study among MLGs isolates within Group 1, according to results of phylogenetic and subpopulation genetics analyses. The four SPs all appear to have an epidemic population structure. In previous studies, the ITS genotype Macaque3 was mostly found in NHPs, and only occasionally in dogs [8-10, 26, 51]. Therefore, the clustering of the host-adapted ITS genotype Macaque3 in SP3 and SP4 suggests the NHP-specific nature of these two subpopulations. Both potentially zoonotic and host-adapted subpopulations have been identified in Group 1 in previous studies. The former included MLGs of ITS genotypes Type IV, D, Peru8, WL11, and Peru11 in humans, NHPs, pigs and 

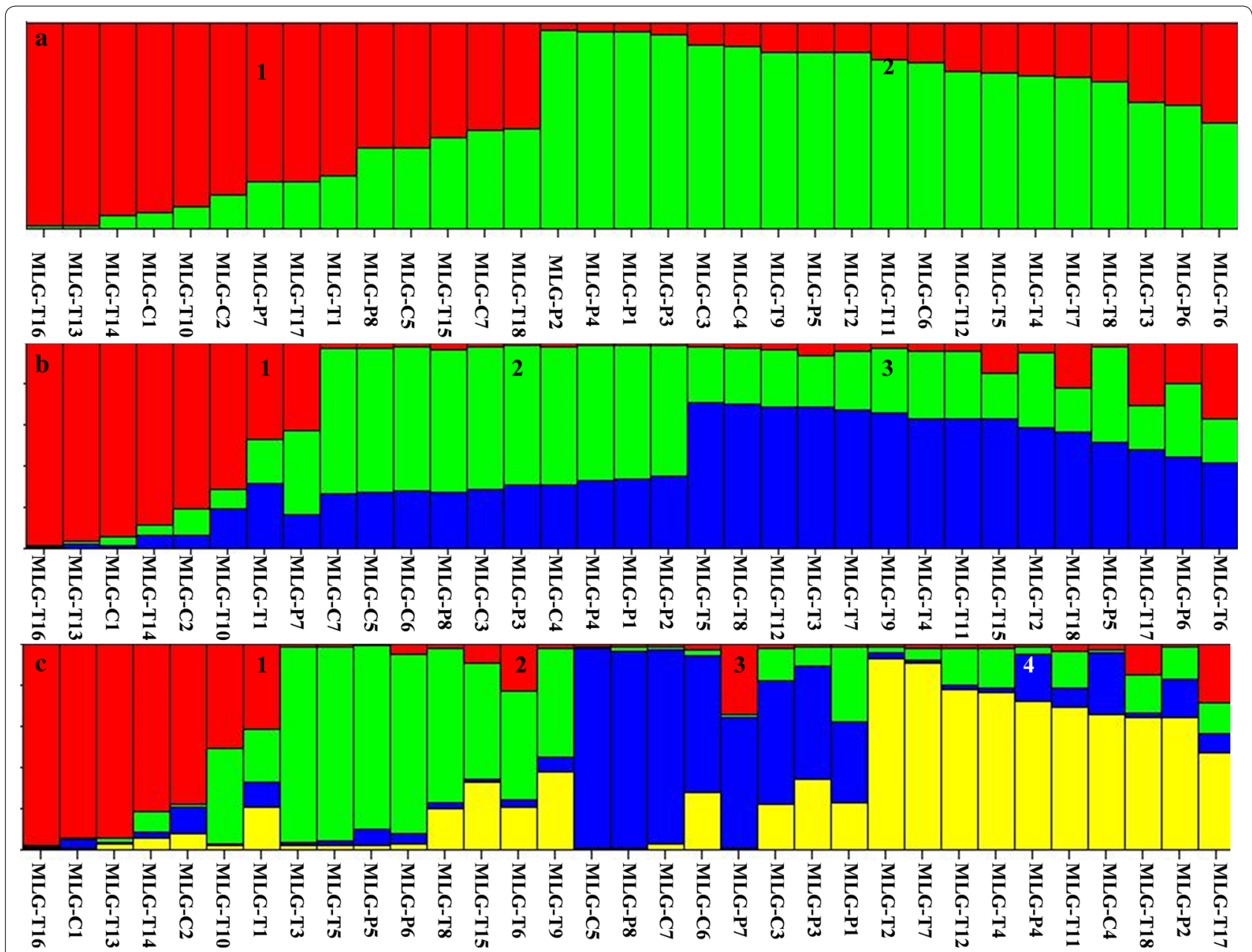

Fig. 3 Subpopulation structure of 33 Enterocytozoon bieneusi MLGs. Different subpopulation patterns are shown depending on the setting of $K$ values. a The generation of two subpopulations using $K=2$. b The presence of three subpopulations using $K=3$. $\mathbf{c}$ The appearance of four subpopulations using $K=4$

companion animals, whereas the latter included MLGs of ITS genotypes A in humans, EbpA and CS-4 in pigs, and Macaque3 in NHPs $[1,15,21,36,52,53]$. Therefore, SP3 and SP4 including MLGs of Macaque3 were potentially NHPs-adapted subpopulations, while SP1 and SP2 only including MLGs of ITS genotype Type IV and Peru8 were potentially zoonotic subpopulations.

\section{Conclusions}

A much higher genetic diversity was revealed in E. bieneusi isolates from crab-eating macaques by multilocus sequence typing (33 MLGs) than by ITS genotyping (three ITS genotypes). This appears to have resulted from genetic recombination among common ITS genotypes despite an overall clonal population structure in E. bieneusi. Nevertheless, the MLGs generated from the analysis have formed four subpopulations with different host ranges and an epidemic population structure. These results provide data on the population and subpopulation structure of E. bieneusi in NHPs, and would improve our perception of E. bieneusi epidemiology in these animals.

\section{Abbreviations}

ITS: internal transcribed spacer; LD: linkage disequilibrium; LE: linkage equilibrium; ML: maximum likelihood; MLG: multi-locus genotype; NHP: non-human primate; PCR: polymerase chain reaction; Rms: recombination events; SP: subpopulation; UPGMA: un-weighted pair group mean average.

\section{Acknowledgements}

We thank the farm owner and staff for their assistance in sample collection during this study.

\section{Authors' contributions}

YF and LX conceived and designed the experiments. LC performed the experiments. LC, NL and YG analyzed the data. LC, YF and LX wrote the paper. All authors read and approved the final manuscript. 


\section{Funding}

This study was supported by the National Key R\&D Program of China (2017YFC1601206) and the National Natural Science Foundation of China (31820103014).

\section{Availability of data and materials}

The data supporting the conclusions of this article are included within the article. Representative nucleotide sequences generated in this study were deposited in the GenBank database under the accession numbers MT137078-MT137095

\section{Ethics approval and consent to participate}

This study was approved by the Research Ethics Committee of the East China University of Science and Technology, with the approval number of 2015018 on 3rd January 2015. Permission for collecting fecal specimens was obtained from the managers of the farms. Animals were kept according to the Animal Ethics Procedures and Guidelines of the People's Republic of China.

\section{Consent for publication}

Not applicable.

\section{Competing interests}

The authors declare that they have no competing interests.

\section{Author details}

${ }^{1}$ State Key Laboratory of Bioreactor Engineering, School of Resource and Environmental Engineering, East China University of Science and Technology, Shanghai 200237, China. ${ }^{2}$ Key Laboratory of Zoonosis of Ministry of Agriculture, College of Veterinary Medicine, South China Agricultural University, Guangzhou 510642, China. ${ }^{3}$ Guangdong Laboratory for Lingnan Modern Agriculture, Guangzhou 510642, China. ${ }^{4}$ Key Laboratory of Tropical Biological Resources of Ministry of Education, School of Life and Pharmaceutical Sciences, Hainan University, Haikou 570228, Hainan, China.

Received: 31 December 2019 Accepted: 27 March 2020

Published online: 08 April 2020

\section{References}

1. Santin M, Fayer R. Microsporidiosis: Enterocytozoon bieneusi in domesticated and wild animals. Res Vet Sci. 2011;90:363-71.

2. Desportes I, Le Charpentier Y, Galian A, Bernard F, Cochand-Priollet B, Lavergne A, et al. Occurrence of a new microsporidan: Enterocytozoon bieneusi n.g., n. sp., in the enterocytes of a human patient with AIDS. J Protozool. 1985;32:250-4.

3. Tavalla M, Mardani-Kateki M, Abdizadeh R, Nashibi R, Rafie A, Khademva$\tan$ S. Molecular identification of Enterocytozoon bieneusi and Encephalitozoon spp. in immunodeficient patients in Ahvaz, Southwest of Iran. Acta Trop. 2017:172:107-12.

4. Didier ES, Weiss LM. Microsporidiosis: not just in AIDS patients. Curr Opin Infect Dis. 2011:24:490-5.

5. Lobo ML, Xiao L, Antunes F, Matos O. Microsporidia as emerging pathogens and the implication for public health: a 10-year study on HIVpositive and -negative patients. Int J Parasitol. 2012;42:197-205.

6. Li W, Feng Y, Santin M. Host specificity of Enterocytozoon bieneusi and public health implications. Trends Parasitol. 2019;35:436-51.

7. Matos O, Lobo ML, Xiao L. Epidemiology of Enterocytozoon bieneusi infection in humans. J Parasitol Res. 2012;2012:981424.

8. Chen L, Zhao J, Li N, Guo Y, Feng Y, Feng Y, et al. Genotypes and public health potential of Enterocytozoon bieneusi and Giardia duodenalis in crab-eating macaques. Parasites Vectors. 2019;12:254.

9. Karim MR, Dong H, Li T, Yu F, Li D, Zhang L, et al. Predomination and new genotypes of Enterocytozoon bieneusi in captive nonhuman primates in zoos in China: high genetic diversity and zoonotic significance. PLOS ONE. 2015;10:e0117991.

10. Yang H, Lin Y, Li Y, Song M, Lu Y, Li W. Molecular characterization of Enterocytozoon bieneusi isolates in laboratory macaques in north China: zoonotic concerns. Parasitol Res. 2017;116:2877-82.
11. Zhong Z, Li W, Deng L, Song Y, Wu K, Tian Y, et al. Multilocus genotyping of Enterocytozoon bieneusi derived from nonhuman primates in southwest China. PLoS ONE. 2017;12:e0176926.

12. Mynarova A, Foitova I, Kvac M, Kvetonova D, Rost M, Morrogh-Bernard $\mathrm{H}$, et al. Prevalence of Cryptosporidium spp., Enterocytozoon bieneusi, Encephalitozoon spp. and Giardia intestinalis in wild, semi-wild and captive orangutans (Pongo abelii and Pongo pygmaeus) on Sumatra and Borneo, Indonesia. PLoS ONE. 2016;11:e0152771.

13. Sak B, Petrzelkova KJ, Kvetonova D, Mynarova A, Pomajbikova K, Modry D, et al. Diversity of microsporidia, Cryptosporidium and Giardia in mountain gorillas (Gorilla beringei beringei) in Volcanoes National Park, Rwanda. PLoS ONE. 2014;9:e109751.

14. Li W, Kiulia NM, Mwenda JM, Nyachieo A, Taylor MB, Zhang X, et al. Cyclospora papionis, Cryptosporidium hominis, and human-pathogenic Enterocytozoon bieneusi in captive baboons in Kenya. J Clin Microbiol. 2011:49:4326-9.

15. Li W, Xiao L. Multilocus sequence typing and population genetic analysis of Enterocytozoon bieneusi: host specificity and its impacts on public health. Front Genet. 2019;10:307.

16. Li W, Feng Y, Zhang L, Xiao L. Potential impacts of host specificity on zoonotic or interspecies transmission of Enterocytozoon bieneusi. Infect Genet Evol. 2019:75:104033.

17. Ye J, Xiao L, Ma J, Guo M, Liu L, Feng Y. Anthroponotic enteric parasites in monkeys in public park, China. Emerg Infect Dis. 2012;18:1640-3.

18. Zhang $X$, Wang Z, Su Y, Liang $X$, Sun $X$, Peng S, et al. Identification and genotyping of Enterocytozoon bieneusi in China. J Clin Microbiol. 2011:49:2006-8.

19. Du S, Zhao G, Shao J, Fang Y, Tian G, Zhang L, et al. Cryptosporidium spp., Giardia intestinalis, and Enterocytozoon bieneusi in captive non-human primates in Qinling mountains. Korean J Parasitol. 2015;53:395-402.

20. Sak B, Petrzelkova KJ, Kvetonova D, Mynarova A, Shutt KA, Pomajbikova $\mathrm{K}$, et al. Long-term monitoring of microsporidia, Cryptosporidium and Giardia infections in western lowland gorillas (Gorilla gorilla gorilla) at different stages of habituation in Dzanga Sangha Protected Areas, Central African Republic. PLoS ONE. 2013;8:e71840.

21. Karim MR, Wang R, Dong H, Zhang L, Li J, Zhang S, et al. Genetic polymorphism and zoonotic potential of Enterocytozoon bieneusi from nonhuman primates in China. Appl Environ Microbiol. 2014;80:1893-8.

22. Wang S, Wang R, Fan X, Liu T, Zhang L, Zhao G. Prevalence and genotypes of Enterocytozoon bieneusi in China. Acta Trop. 2018:183:142-52.

23. Lores B, Lopez-Miragaya I, Arias C, Fenoy S, Torres J, del Aguila C. Intestinal microsporidiosis due to Enterocytozoon bieneusi in elderly human immunodeficiency virus-negative patients from Vigo, Spain. Clin Infect Dis. 2002;34:918-21.

24. Wang L, Zhang H, Zhao X, Zhang L, Zhang G, Guo M, et al. Zoonotic Cryptosporidium species and Enterocytozoon bieneusi genotypes in HIV-positive patients on antiretroviral therapy. J Clin Microbiol. 2013;51:557-63.

25. Santin M, Fayer R. Enterocytozoon bieneusi genotype nomenclature based on the internal transcribed spacer sequence: a consensus. J Eukaryot Microbiol. 2009:56:34-8.

26. Karim MR, Dong H, Yu F, Jian F, Zhang L, Wang R, et al. Genetic diversity in Enterocytozoon bieneusi isolates from dogs and cats in China: host specificity and public health implications. J Clin Microbiol. 2014;52:3297-302.

27. Luo R, Xiang L, Liu H, Zhong Z, Liu L, Deng L, et al. First report and multilocus genotyping of Enterocytozoon bieneusi from Tibetan pigs in southwestern China. Parasite. 2019;26:24.

28. Zou Y, Hou J, Li F, Zou F, Lin R, Ma J, et al. Prevalence and genotypes of Enterocytozoon bieneusi in pigs in southern China. Infect Genet Evol. 2018;66:52-6.

29. Tang C, Cai M, Wang L, Guo Y, Li N, Feng Y, et al. Genetic diversity within dominant Enterocytozoon bieneusi genotypes in pre-weaned calves. Parasites Vectors. 2018;11:170.

30. Tian G, Zhao G, Du S, Hu X, Wang H, Zhang L, et al. First report of Enterocytozoon bieneusi from giant pandas (Ailuropoda melanoleuca) and red pandas (Ailurus fulgens) in China. Infect Genet Evol. 2015;34:32-5.

31. Deng L, Li W, Yu X, Gong C, Liu X, Zhong Z, et al. First report of the human-pathogenic Enterocytozoon bieneusi from red-bellied tree squirrels (Callosciurus erythraeus) in Sichuan, China. PLoS ONE. 2016;11:e0163605. 
32. Feng Y, Li N, Dearen T, Lobo ML, Matos O, Cama V, et al. Development of a multilocus sequence typing tool for high-resolution genotyping of Enterocytozoon bieneusi. Appl Environ Microbiol. 2011;77:4822-8.

33. Li W, Cama V, Feng Y, Gilman RH, Bern C, Zhang X, et al. Population genetic analysis of Enterocytozoon bieneusi in humans. Int J Parasitol. 2012;42:287-93.

34. Desoubeaux G, Nourrisson C, Moniot M, De Kyvon MA, Bonnin V, De La Bretonniere ME, et al. Genotyping approach for potential common source of Enterocytozoon bieneusi infection in hematology unit. Emerg Infect Dis. 2019;25:1625-31.

35. Widmer G, Dilo J, Tumwine JK, Tzipori S, Akiyoshi DE. Frequent occurrence of mixed Enterocytozoon bieneusi infections in humans. Appl Environ Microbiol. 2013;79:5357-62.

36. Karim MR, Wang R, He X, Zhang L, Li J, Rume Fl, et al. Multilocus sequence typing of Enterocytozoon bieneusi in nonhuman primates in China. Vet Parasitol. 2014;200:13-23.

37. Li W, Cama V, Akinbo FO, Ganguly S, Kiulia NM, Zhang X, et al. Multilocus sequence typing of Enterocytozoon bieneusi: lack of geographic segregation and existence of genetically isolated sub-populations. Infect Genet Evol. 2013;14:111-9.

38. Rozas J, Sanchez-DelBarrio JC, Messeguer X, Rozas R. DnaSP, DNA polymorphism analyses by the coalescent and other methods. Bioinformatics. 2003;19:2496-7.

39. Excoffier $L$, Lischer HE. Arlequin suite ver 3.5: a new series of programs to perform population genetics analyses under Linux and Windows. Mol Ecol Resour. 2010;10:564-7.

40. Haubold B, Hudson RR. LIAN 3.0: detecting linkage disequilibrium in multilocus data. Linkage Analysis. Bioinformatics. 2000;16:847-8.

41. Falush D, Stephens M, Pritchard JK. Inference of population structure using multilocus genotype data: linked loci and correlated allele frequencies. Genetics. 2003;164:1567-87.

42. Gui B, Zou Y, Chen Y, Li F, Jin Y, Liu M, et al. Novel genotypes and multilocus genotypes of Enterocytozoon bieneusi in two wild rat species in China: potential for zoonotic transmission. Parasitol Res. 2020;119:283-90.

43. Zhang N, Wu R, Ji T, Cui L, Cao H, Li D, et al. Molecular detection, multilocus genotyping, and population genetics of Enterocytozoon bieneusi in pigs in southeastern China. J Eukaryot Microbiol. 2020;67:107-14.

44. Ma Y, Zou Y, Liu Q, Xie S, Li R, Zhu X, et al. Prevalence and multilocus genotypes of Enterocytozoon bieneusi in alpacas (Vicugna pacos) in Shanxi Province, northern China. Parasitol Res. 2019;118:3371-5.
45. Zhao G, Du S, Wang H, Hu X, Deng M, Yu S, et al. First report of zoonotic Cryptosporidium spp., Giardia intestinalis and Enterocytozoon bieneusi in golden takins (Budorcas taxicolor bedfordi). Infect Genet Evol. 2015;34:394-401.

46. Deng L, Li W, Zhong Z, Gong C, Liu X, Huang X, et al. Molecular characterization and multilocus genotypes of Enterocytozoon bieneusi among horses in southwestern China. Parasites Vectors. 2016;9:561.

47. Li W, Wan Q, Yu Q, Yang Y, Tao W, Jiang Y, et al. Genetic variation of miniand microsatellites and a clonal structure in Enterocytozoon bieneusi population in foxes and raccoon dogs and population differentiation of the parasite between fur animals and humans. Parasitol Res. 2016;115:2899-904.

48. Wang X, Wang R, Ren G, Yu Z, Zhang L, Zhang S, et al. Multilocus genotyping of Giardia duodenalis and Enterocytozoon bieneusi in dairy and native beef (Qinchuan) calves in Shaanxi Province, northwestern China. Parasitol Res. 2016;115:1355-61.

49. Li W, Song Y, Zhong Z, Huang X, Wang C, Li C, et al. Population genetics of Enterocytozoon bieneusi in captive giant pandas of China. Parasites Vectors. 2017;10:499.

50. Wan Q, Xiao L, Zhang X, Li Y, Lu Y, Song M, et al. Clonal Evolution of Enterocytozoon bieneusi populations in swine and genetic differentiation in subpopulations between isolates from swine and humans. PLoS Negl Trop Dis. 2016;10:e0004966.

51. Zhao W, Zhou H, Jin H, Sun L, Li P, Liu M, et al. Genotyping of Enterocytozoon bieneusi among captive long-tailed macaques (Macaca fascicularis) in Hainan Province: high genetic diversity and zoonotic potential. Acta Trop. 2020;201:105211.

52. Thellier M, Breton J. Enterocytozoon bieneusi in human and animals, focus on laboratory identification and molecular epidemiology. Parasite. 2008;15:349-58.

53. Li D, Zheng S, Zhou C, Karim MR, Wang L, Wang H, et al. Multilocus typing of Enterocytozoon bieneusi in pig reveals the high prevalence, zoonotic potential, host adaptation and geographical segregation in China. J Eukaryot Microbiol. 2019;66:707-18.

\section{Publisher's Note}

Springer Nature remains neutral with regard to jurisdictional claims in published maps and institutional affiliations.
Ready to submit your research? Choose BMC and benefit from:

- fast, convenient online submission

- thorough peer review by experienced researchers in your field

- rapid publication on acceptance

- support for research data, including large and complex data types

- gold Open Access which fosters wider collaboration and increased citations

- maximum visibility for your research: over $100 \mathrm{M}$ website views per year

At BMC, research is always in progress.

Learn more biomedcentral.com/submissions 